\title{
Efficient Gene Transfer into Chicken Gonads by Combining Transposons with Polyethylenimine
}

\author{
Saisai Wang ${ }^{1}$, Yali Wang ${ }^{1}$, Dan Shen ${ }^{1}$, Li Zhang ${ }^{1}$, Songlei Xue ${ }^{1}$, Hengmi Cui ${ }^{1}$, Chengyi Song ${ }^{1} \&$ Bo Gao ${ }^{1}$ \\ ${ }^{1}$ Joint International Research Laboratory of Agriculture and Agri-product Safety, Institute of Epigenetics \& \\ Epigenomics, College of Animal Science \& Technology, Yangzhou University, Yangzhou, Jiangsu, China \\ Correspondence: Bo Gao, Joint International Research Laboratory of Agriculture and Agri-product Safety, \\ Institute of Epigenetics \& Epigenomics, College of Animal Science \& Technology, Yangzhou University, \\ Yangzhou, Jiangsu 225009, China. Tel: 86-514-8797-9034. Fax: 86-514-8735-0440. E-mail: bgao@yzu.edu.cn
}

Received: July 23, 2016

doi:10.5539/jas.v8n10p63
Accepted: August 17, $2016 \quad$ Online Published: September 15, 2016

URL: http://dx.doi.org/10.5539/jas.v8n10p63

\begin{abstract}
Transposon mediated transfection is a promising, safe, and convenient way to generate transgenic chicken compared with virus-mediated technology and the in vitro modification of primordial germ cells (PGCs). To establish a simple method for in vivo transfection of chicken PGCs, we applied four different transposon systems ( $\mathrm{PB}, \mathrm{SB}, \mathrm{Tol} 2$, and $\mathrm{ZB}$ ) to investigate the gene transfer efficiency of chicken gonads via direct injection of a mixture of transposon and transposase plasmids and transfection reagent (polyethylenimine, PEI) into the subgerminal cavity of Hamburger and Hamilton stage 2-3 chick embryos. We also compared the effect of the amount of plasmids injected on the gene transfer efficiency of chicken gonads. We found that over $70 \%$ of the gonads were green fluorescent protein (GFP)-positive across all four transposon groups, and that the proportion of GFP-positive gonads was not significantly different between different transposons. Some GFP positive cells in gonads were confirmed as germ cells by co-labeling with the germ cell specific antibody. We also found that the proportions of GFP-positive gonads decreased significantly with a decrease of plasmid dose from $100 \mathrm{ng}$ to 20 or $50 \mathrm{ng}$. Here we revealed that a combination of transposons with PEI is a simple and efficient method for gene transfer into chicken gonads and able to transfect PGCs in vivo that could be used for the production of transgenic chickens.
\end{abstract}

Keywords: transposon, primordial germ cells, polyethylenimine, transfection efficiency, chicken, transgenesis

\section{Introduction}

The chick embryo, as a model system, has many applications in developmental biology research (Rashidi \& Sottile, 2009; Vergara \& Canto-Soler, 2012) and as a bioreactor for pharmaceutical proteins (Lillico et al., 2005). This model becomes increasingly popular because of peculiar advantages, such as a short incubation period, the accessibility of embryos, and the availability of experimental embryology (Stern, 2005). According to contemporary transgenic methods, viral injection has proven to have the highest efficiency in birds (McGrew et al., 2004), and has been used to generate transgenic hens that synthesize functional recombinant pharmaceutical proteins in a tightly regulated, tissue-specific manner (Lillico et al., 2007); however, viral integration methods have size and sequence restraints (Scott et al., 2010). The most important effect is that transgene expression with viral vector may be silenced in the germline. Because of these potential drawbacks, transposons have become an alternative to viruses for integration and expression of transgenes. Transgenic offspring generated by transposon vectors (such as Minos, Tol1, Tol2, piggyBac [PB], and sleeping beauty [SB]) have been achieved in invertebrates, fish, and mammals (Dupuy et al., 2002; Kawakami et al., 2000; Sasakura et al., 2003; Sumiyama et al., 2010; Wilber et al., 2006). Three commonly used transposons-PB, SB, and Tol2-have also been utilized to transfer foreign genes to the avian genome. The SB transposon system was the first used to try and increase the transgene efficiency in chicken and turkey cells (Kong et al., 2008), while a germ-line-competent chicken PGC line was developed with the PB transposon and transposase, and both of the efficiency of transgenesis and the expression level of transgene were improved with these modified PGCs (Park \& Han, 2012). A transgenic chick can be generated with the PGCs modified in vitro using both PB and Tol2 transposons (Macdonald et al., 2012). Lu et al. (2015) first demonstrated that a PB transposon mediated the insertion of a transgene into chicken embryos during developmental stages. Later, both PB and Tol2 were shown effective for chimera production and 
germline transgenesis by direct injection into the vasculature of developing chick embryos or the subgerminal cavity of newly laid eggs (Liu et al., 2012; Tyack et al., 2013). In addition, transfection reagents such as Lipofectamine 2000 and jetPEI, combined with transposons, have also been applied in the direct injection of chick embryos (Jordan et al., 2014; Tyack et al., 2013), and were proven to be effective and promising for chicken transgenesis.

Although all three transposons (PB, SB, and Tol2) have been tested in avian models, to our knowledge, there is no report on the parallel comparison of the efficiency of these transposons using the same protocol. In the present study, we investigated the gene transfer efficiency into the chicken gonads mediated with three classic transposons ( $\mathrm{PB}, \mathrm{SB}$, and $\mathrm{To} 2 \mathrm{2}$ ) and one new transposon (ZB), which was isolated in our laboratory, by direct injection into the subgerminal cavity of early chicken embryos. We also tested PEI to deliver DNA. PEI was the second polymeric transfection agent discovered after poly-L-lysine (Boussif et al., 1995), and has been used to deliver DNA and RNA both in vivo and in vitro (Lu et al., 2015; Lungwitz et al., 2005; Morishita et al., 2015; Sramkova et al., 2014). This research aimed to establish a simple and efficient method for PGC transfection by direct injection into embryos and to select the best transposon system for further application in avian transgenesis.

\section{Materials and Methods}

\subsection{Animals}

Newly fertilized eggs of Rucao chickens were purchased from the Institute of Poultry Science, Chinese Academy of Agricultural Science. The eggs were kept in an incubator at $37.8^{\circ} \mathrm{C}$ for $10 \mathrm{~h}$, and embryos from Hamburger and Hamilton (HH) stage 2 to $\mathrm{HH} 3$ were used for gene transfection in vivo. The protocol for animal use was approved in accordance with the University Council on Animal Care guidelines.

\subsection{Plasmid DNA}

Two vector systems harboring four transposons were used to transfect the PGCs. One vector system constructed in our laboratory is a trinity of $\mathrm{PB}, \mathrm{SB}$, and Tol2 transposons named pT3-CAG-GFP. This system consists of the terminal inverted repeat sequences (TIRs) of three transposons and a green fluorescence protein (GFP) expression box, including the chicken $\beta$-actin promoter, GFP ORF, and rabbit $\beta$-globin poly A (Figure 1A). The other vector system contains the same GFP expression box, but is flanked with the TIRs of a new Tcl-like transposon that was isolated from zebrafish in our laboratory (named $\mathrm{ZB}$, unpublished data) and named pZB-CAG-GFP (Figure 1B). The PB, SB, ZB, and Tol2 transposase ORF were cloned downstream of the CMV promoter and located upstream of the rabbit $\beta$-globin poly $A$, respectively. Those vectors were named pCMV-PB, pCMV-SB, pCMV-Tol2, and pCMV-ZB (Figure 1C). Both of the PB and SB transposases (pCMV-PB and pCMV-SB) are new versions of vectors that were recently optimized (Mates et al., 2009; Yusa et al., 2011).

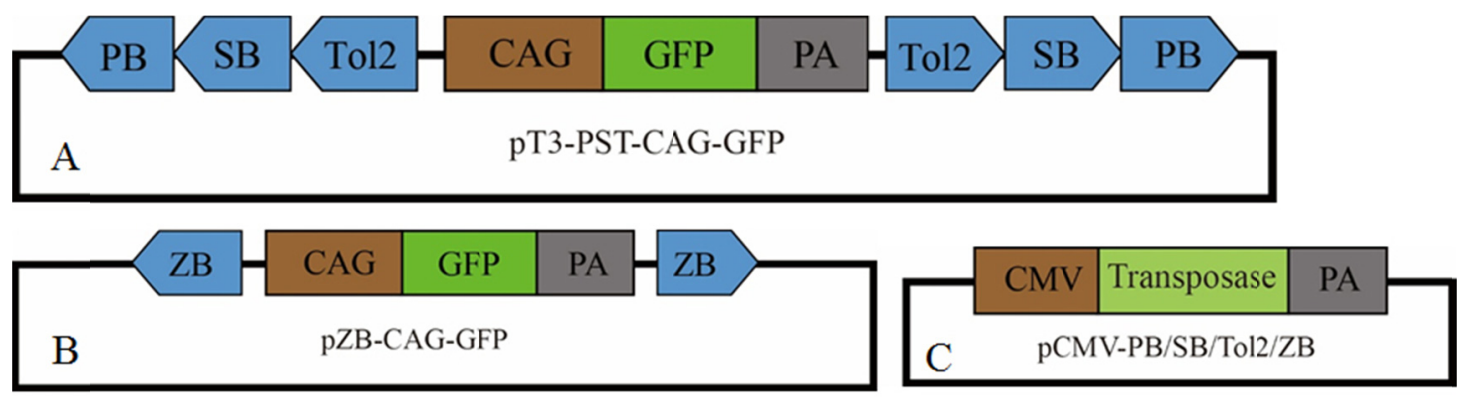

Figure 1. Structure of vectors

Note. A: pT3-CAG-GFP; B: pZB-CAG-GFP; C: pCMV-PB, pCMV-SB, pCMV-Tol2, and pCMV-ZB.

\subsection{Formulation of a Plasmid Complex for Microinjection}

We mixed a transposon plasmid with transposase plasmid in a 2:1 ratio, and then mixed PEI with plasmid in a 2:1 ratio. The plasmid and PEI complex was prepared according to the polyethylenimine manufacturer's instructions (PEI, $25 \mathrm{kDa}$ branched PEI, Sigma-Aldrich). Briefly, transposon vector (pT3-CAG-GFP or 
pZB-CAG-GFP) and transposase vector (pCMV-PB, pCMV-SB100X, pCMV-Tol2, or pCMV-ZB) were mixed at $2: 1$, respectively, and incubated at room temperature for $5 \mathrm{~min}$. Then, an appropriate amount of stock PEI (10 $\mu \mathrm{g} / \mu \mathrm{L}$ ) was added to the DNA mixture at 2:1 and incubated for $15 \mathrm{~min}$ at room temperature. Finally, sterile Trypan blue was added to the mixture at final concentration of $10 \%$ (vol $/ \mathrm{vol})$ and dissolved completely. The final DNA concentrations of 100,50 , or $20 \mathrm{ng} / \mu \mathrm{L}$ were used for injection. This mixture is stable at room temperature for several hours.

\subsection{Microinjection of Chick Embryos}

PGCs are enriched in the transparent region of chick embryos at HH stage 2-3 (6-12 h of incubation) (Kang et al., 2015), which suggests that this stage is an appropriate time for PGC transfection. A window was cut in the pointed end of a recipient egg to allow access to the HH stage 2-3 embryo. The prepared plasmid-PEI complex $(1 \mu \mathrm{L})$ was injected into the central part of subgerminal cavity using a micropipette $(90 \mathrm{~mm}$, Japan) and a microinjector (Tritech Research, America). The injection pipette was drawn from a Narishige glass tube with an inner diameter of $30 \mu \mathrm{m}$ at the tip. After injection, the opening in the egg was sealed with a type of adhesive medical rubberized fabric plaster tape. The eggs were incubated at $37.8^{\circ} \mathrm{C}$ and $60 \%$ relative humidity with a rocking motion every $2 \mathrm{~h}$ through a $90^{\circ}$ angle for 18 days, after which they were further incubated at $37^{\circ} \mathrm{C}$ and $70 \%$ relative humidity without rocking until they hatched.

\subsection{Detection of Green Fluorescence}

To assess the success of the technique, all embryos were hatched for 21 days, then eight organs, including the heart, liver, spleen, lung, kidney, brain, gonads, and intestine, were dissected to detect the GFP signal under a fluorescence microscope. At the same time, the viability data at embryo day 7 (ED 7) and ED 14 and the hatching rate at ED 21 were collected.

\subsection{Immunohistochemistry}

We used immunostaining of chicken Vasa homologue gene $(\mathrm{CVH})$ to identify GFP-positive germ cells. Briefly, dissected gonads were dissociated using trypsin and fixed in $4 \%$ paraformaldehyde for $10 \mathrm{~min}$. The resulting cells were centrifuged for 5 min and the supernatant was discarded. The cells were washed two times in phosphate-buffered saline (PBS) and nonspecific binding sites were blocked with $1 \%$ bovine serum albumin in PBST for $30 \mathrm{~min}$, then centrifuged for $5 \mathrm{~min}$ per wash, and the supernatant discarded. The cells were subsequently incubated with the primary rabbit polyclonal anti-DDX4 at 1:100 (Beijing Biosynthesis Biotechnology Co.) overnight at $4{ }^{\circ} \mathrm{C}$ and washed three times in PBST for 5 min per wash. The cells were then incubated with secondary Cy3-conjugated goat anti-rabbit antibody (Beijing Biosynthesis Biotechnology Co.) at 1:200 for $1 \mathrm{~h}$ at room temperature and then washed three times with PBST for 5 min per wash. Finally, the cells were stained with DAPI $(1 \mu \mathrm{g} / \mathrm{mL})$ and visualized using a fluorescence microscope.

\subsection{Statistical Analysis}

A chi-square test was used to determine the significance of differences between the GFP-positive proportion of gonads, GFP-positive proportion of embryos, viability at ED 7 and ED 14, and hatching rates at ED 21 between groups. $P<0.05$ was considered significant.

\section{Results}

\subsection{Efficient Gene Transfer into Chicken Gonads Using Different Transposons}

Four transposons systems were used to evaluate the gene transfer efficiency into chicken gonads. The transposon and transposase plasmids were combined and formulated with PEI to produce the injection mixture. Then, the mixture was injected into the subgerminal cavity of $\mathrm{HH} 2-3$ embryos to transfect the PGCs, as previously described. All injected embryos were incubated for 21 days. Then, the GFP-positive proportions of gonads and embryos, viability, and hatching proportions were analyzed. High gene transfer efficiencies into the gonads were obtained (Table 1). Most gonads of injected embryos were GFP-positive in all four transposon groups. The GFP-positive proportions of gonads were $70.97 \%, 78.26 \%, 77.42 \%$, and $72.00 \%$ in PB, SB, Tol2, and ZB, respectively. We found no significant difference between the four transposons $(P>0.05)$ based on a chi-square test. The GFP signals were detected in diverse tissues, including gonads (Figure 2A), spleen (Figure 2B), intestine (Figure 2C), and brain (Figure 2D). Green fluorescence was observed extensively throughout the gonads (Figure 2A). We found that the proportion of GFP-positive embryos (including embryos with any GFP-positive tissue) was about $80 \%$ for all transposon groups. No significant differences were found between transposons $(P>0.05)$. 
Table 1. Hatching rates and transfection efficiency of manipulated eggs

\begin{tabular}{llllll}
\hline Group & $\begin{array}{l}\text { Viability } \\
\text { at ED 7 }(\%)\end{array}$ & $\begin{array}{l}\text { Viability } \\
\text { at ED 14 }(\%)\end{array}$ & $\begin{array}{l}\text { Hatching rate } \\
\text { at ED 21 }(\%)\end{array}$ & $\begin{array}{l}\text { Proportion of GFP } \\
\text { embryos }(\%)\end{array}$ & $\begin{array}{l}\text { Proportion of GFP }^{+} \\
\text {gonads }(\%)\end{array}$ \\
\hline PB & $75.56(68 / 90)$ & $56.67(51 / 90)^{\mathrm{B}}$ & $34.44(31 / 90)$ & $80.65(25 / 31)$ & $70.97(22 / 31)$ \\
SB & $71.11(64 / 90)$ & $40.00(36 / 90)^{\mathrm{A}}$ & $25.56(23 / 90)$ & $78.26(18 / 23)$ & $78.26(18 / 23)$ \\
TOL2 & $80.90(72 / 89)$ & $58.43(52 / 90)^{\mathrm{B}}$ & $34.83(31 / 89)$ & $80.65(25 / 31)$ & $77.42(24 / 31)$ \\
ZB & $83.13(69 / 83)$ & $68.12(47 / 83)^{\mathrm{B}}$ & $30.12(25 / 83)$ & $80.00(20 / 25)$ & $72.00(18 / 25)$ \\
\hline
\end{tabular}

Note. Various letters in the same row indicate significant differences $(P<0.05)$.

The toxic effect of these transposons combining with PEI was then checked. We found that the viability at ED 7 and the hatching rate at ED 21 were generally similar across the four transposon groups $(P>0.05)$, but the viability at ED14 showed a significant difference between the four $(P<0.05)$ (Table 1). Compared with previous studies (Jordan et al., 2014; Tyack et al., 2013), the viability at ED 7 and ED 14 and the hatching rate at ED 21 (25-35\%) were acceptable.
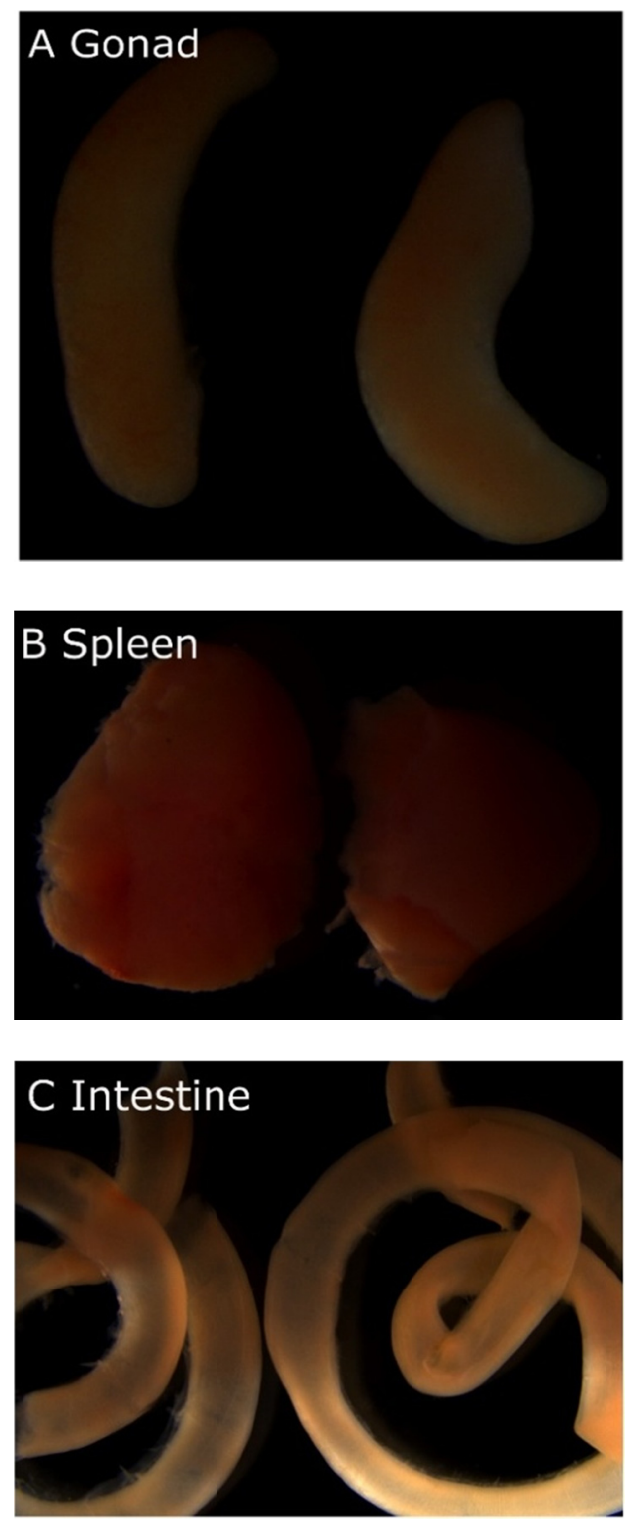
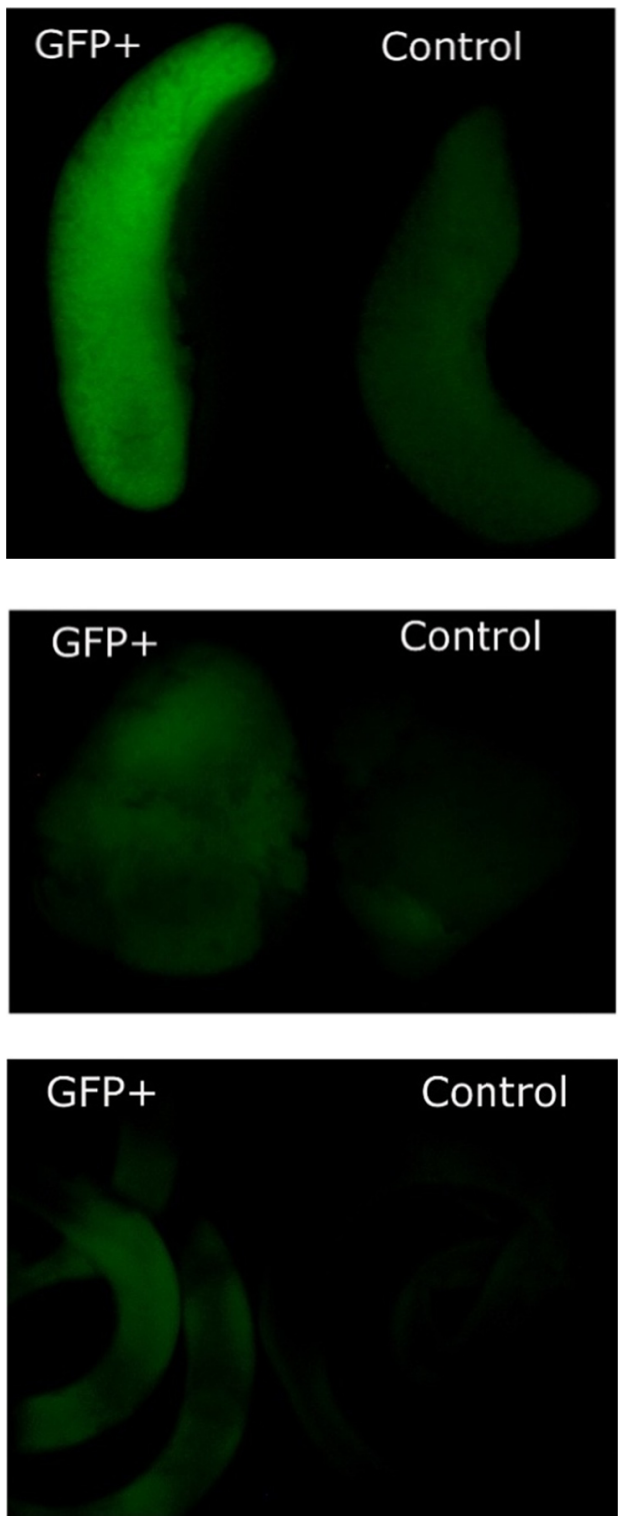

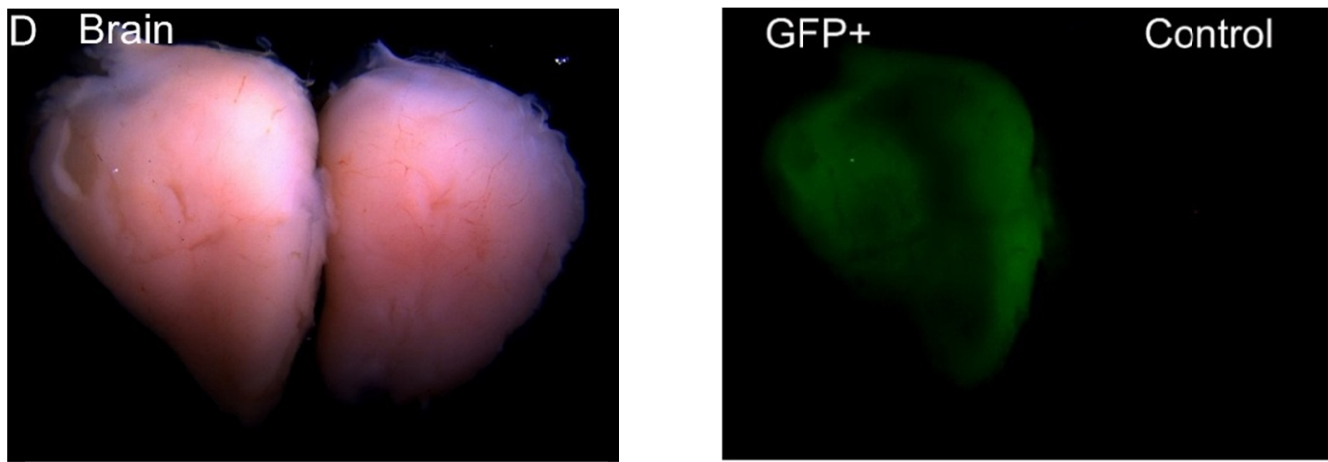

Figure 2. Analysis of fluorescent tissues from directly injected embryos and negative controls

Note. GFP and corresponding bright field images of representative gonads (A), spleen (B), intestine (C), and brain (D).

\subsection{Confirmation of the Ability of Transposons to Transfect Germ Cells Using Immunohistochemistry}

To confirm the ability of these transposons to transfect germ cells, we identified GFP-positive gonad cells by immunohistochemistry for the germ cell-specific antigen, CVH. Some GFP-expressing cells were positive for $\mathrm{CVH}$, which is generally used as a marker for chicken germ cells (Figure 3). We found that we had successfully transfected germ cells in vivo with the transposon-mediated technique.
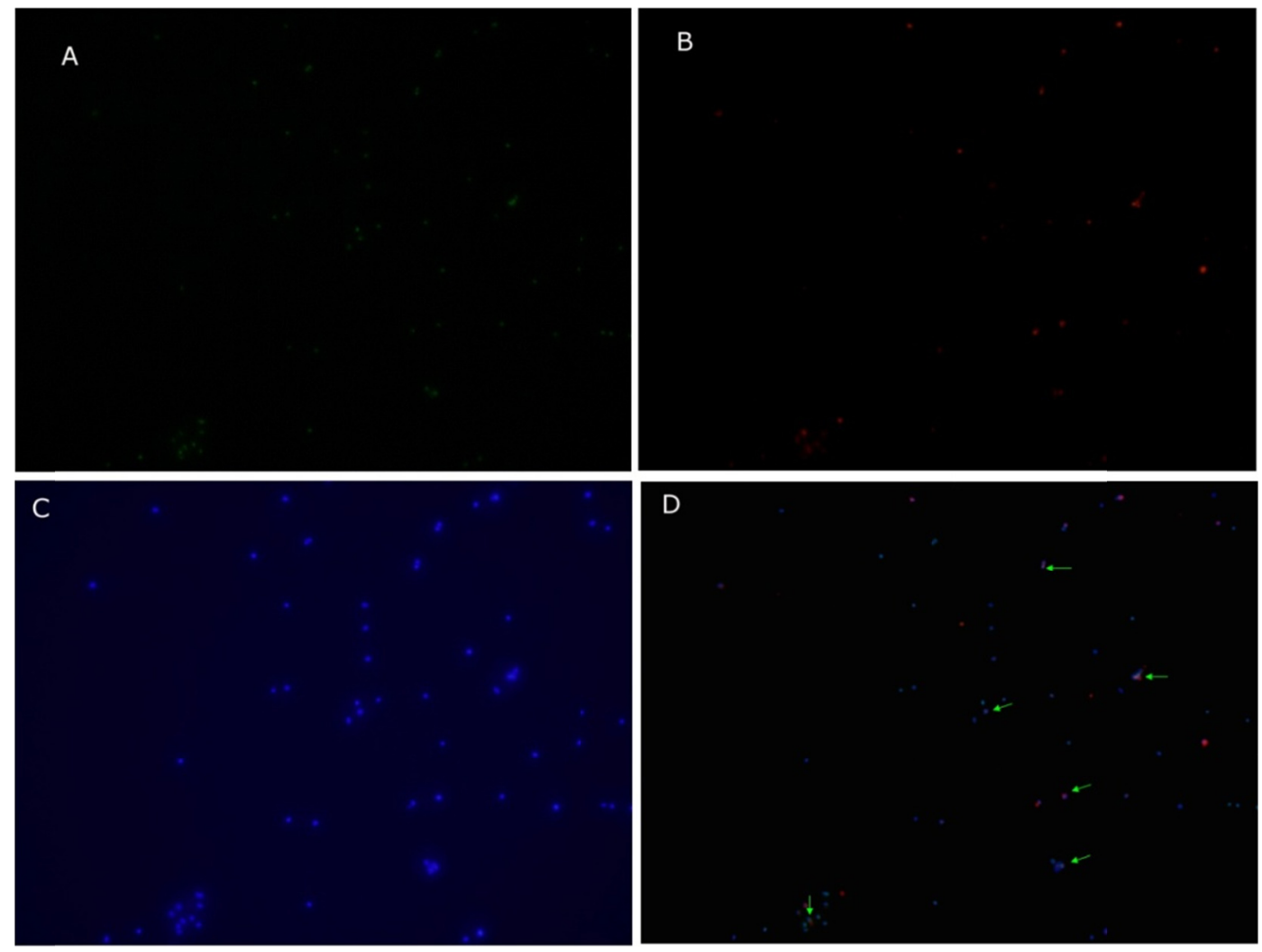

Figure 3. Colocalization of some GFP-positive cells with germ cell-specific antigens in cells from gonads

Note. Cells were observed by fluorescence microscopy (A: GFP; B: anti-CVH; C: DAPI; D: merged images). Arrows indicate colocalization of GFP and immunoreactivity. Magnification $\times 40$. 


\subsection{Effect of the Amount of Plasmid on the in vivo Transfection Efficiency of PGCs}

Next, we examined the effect of the amount of injected plasmid on the in vivo transfection efficiency of PGCs. We decreased the dose of the injected plasmids to $50 \mathrm{ng}$ or $20 \mathrm{ng}$ for each embryo in the SB transposon group. We found that the proportion of GFP-positive gonads and embryos decreased significantly $(P<0.05)$ with the decrease of plasmid dose (Figures $4 \mathrm{~A}$ and $4 \mathrm{~B}$ ), while the viability and hatching rate of the embryos did not increase significantly $(P>0.05)$ (Figures $4 \mathrm{C}, 4 \mathrm{D}$ and $4 \mathrm{E})$.
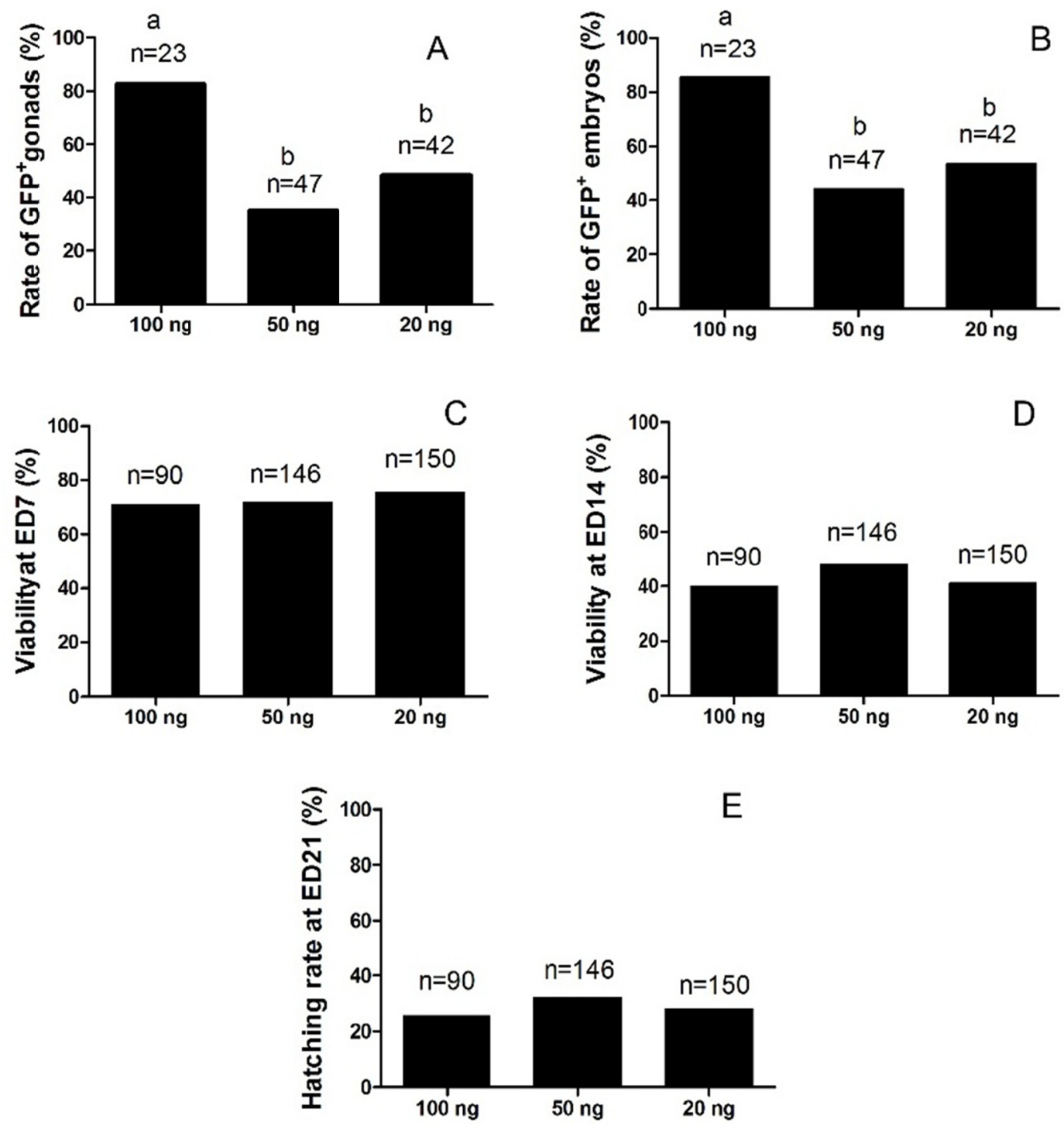

Figure 4. The effect of the amount of plasmid on the proportion of GFP-positive gonads (A) and embryos (B), and the viability and hatching rate of embryos $(\mathrm{C}, \mathrm{D}, \mathrm{E})$

Note. The various letters indicate significant differences $(P<0.05)$.

\section{Discussion}

The current study demonstrated that all four transposons (PB, SB, Tol2, and ZB) combined with the transfection reagent (PEI) were capable of transferring foreign genes into the chicken gonads with similarly high efficiency (over 70\% of the gonads being GFP positive) by direct injection into the subgerminal cavity of HH 2-3 embryos. The gene transfer efficiency of chicken gonads with a high injection dose (100 ng) of plasmid packed with PEI was higher than that of the low dose (20 and $50 \mathrm{ng}$ ). 
Although the transfection of PGCs can be achieved by injecting plasmid DNA and liposome complexes into the bloodstream of stage HH 14 embryos (Watanabe et al., 1994), this method is inefficient and unstable without a transposon medium. Combining transposons with transfection reagents has been shown to be effective for chimera production and germline transgenesis by direct injection into the vasculature of developing chick embryos or stage X embryos (Jordan et al., 2014; Tyack et al., 2013). The combination of Tol2 transposon with Lipofectamine 2000 has been confirmed to transfect the PGCs efficiently in vivo by direct injection into the vasculature of developing chick embryos and to generate stable germ-line transgenic male chickens who passed the transgene onto their offspring (Tyack et al., 2013). That study (Tyack et al., 2013) revealed that all gonads sampled at ED 14 were GFP positive (10 out of 10 embryos), five out of the $11 \mathrm{~F} 0$ male semen were transgene positive, five out of the 419 offspring from the F0 individuals with transgene positive semen were GFP positive, and the transgene rate of their offspring was about $1.5 \%$, which is substantially lower than that using lentiviral vectors (4-45\%) (McGrew et al., 2004).

Other nonviral vehicles, such as PEI, have also been used in combination with the transposon to improve gene transfer efficiency in chickens (Jordan et al., 2014). PEI, a highly water-soluble cationic polymer, has been widely used for nonviral transfection of DNA and RNA in vitro and in vivo (Lu et al., 2015; Lungwitz et al., 2005; Morishita et al., 2015; Sramkova et al., 2014), and the advantage of PEI over other polycations is that it combines strong DNA compaction capacity with an intrinsic endosomolytic activity (Tang \& Szoka, 1997). PEI has two structural forms, linear and branched, with various molecular masses (Fischer et al., 1999; Huh et al., 2007). The linear PEI derivative (jetPEI) combined with the PB transposon, which has been tried in chickens, can transfect multiple chick cell types, including germline stem cells, by direct injection into the vitelline artery of HH stage 23 chick embryos (Jordan et al., 2014). This technique has also been applied for long-term expression of a transgene in chickens by direct injection into stage $\mathrm{X}$ embryos. Stable expression of GFP was seen in multiple tissue types, including the heart, brain, liver, intestine, kidney, and gonads (Jordan et al., 2014). Here we demonstrated that the $25 \mathrm{kDa}$ branched PEI, combined with transposons, can be used to deliver genes into chicken gonads and transfect PGCs efficiently in vivo. To find the most efficient transposon, we compared the transfection efficiency of PGCs across four transposons by screening the GFP-positive gonads. All of these transposons were highly efficient, and no significant difference was observed in the proportion of GFP-positive gonads, which also suggests that all four of these transposons are applicable in chicken transgenesis and that their efficiency is consistent with that previously reported (Tyack et al., 2013). We also found that combining PEI with a high dose (100 ng) of plasmid can generate a high proportion of GFP-positive gonads, while combining PEI with a low dose (20 or $50 \mathrm{ng}$ ) of plasmid can generate a low proportion of GFP-positive gonads, but with a limited increase in the hatching rates and viability of embryos, which indicates that the hatching rates and viability of embryos may be affected mainly by the physical injury of the injection, not the dose of plasmid or transfection reagent. Transposons combined with other techniques, such as electroporation, have also been attempted in chickens (Liu et al., 2012; Sato et al., 2007). However, the gene transfer efficiency, hatching rates, and viability of embryos were substantially lower than that of transposons combined with the transfection reagents reported in this and previous studies (Tyack et al., 2013).

Another common approach to the production of transgenic chickens mediated with transposons is based on PGC culture in vitro. Chicken PGCs are cultured and modified in vitro and then injected into embryos to generate transgenic chickens. Investigators have generated transgenic chickens and offspring efficiently using PB transposons (Macdonald et al., 2012; Park \& Han, 2012). However, as is widely known, it is difficult to isolate many PGCs from embryos, and culturing PGCs in vitro is technically challenging and expensive. Meanwhile, the in vivo transfection of PGCs by combining transposons with a transfection reagent using direct embryo injection is much simpler and more convenient, and the efficiency of PGC transfection is high, which has been proven by this and previous studies (Tyack et al., 2013; Jordan et al., 2014). The protocol described in this study could be used to produce germline transgenics, and may offer a new solution for precision genome manipulation involving CRISPR/Cas9 (Cong et al., 2013), zinc finger nucleases (ZFNs) (Kim et al., 1996), and TALENs (Bedell et al., 2012).

We found that direct embryo injection with the combination of transposons and PEI is a simple and readily adoptable method for the gene transfer into chicken gonads. All four transposons investigated in this study (PB, SB, ZB, Tol2) are highly efficient for the gene transfer into chicken gonads and able to transfect PGCs in vivo, and applicable in chickens. Furthermore, the rates of GFP-positive gonads decreased significantly with the decrease of plasmid dose from $100 \mathrm{ng}$ to $20 \mathrm{or} 50 \mathrm{ng}$. 


\section{Acknowledgements}

This work was supported by the Natural Science Foundation of China (NSFC) [grant number 31200920 and 31671313]; NSFC Major Research Plan [grant number 91540117]; and the Priority Academic Program Development of Jiangsu Higher Education Institutions. We are grateful to Mr. Kehua Wang and Shoufeng Li for providing the fertilized eggs and advise on the embryo hatching.

\section{References}

Bedell, V. M., Wang, Y., Campbell, J. M., Poshusta, T. L., Starker, C. G., Krug, R. G., ... Ekker, S. C. (2012). In vivo genome editing using a high-efficiency TALEN system. Nature, 491, 114-118. http://dx.doi.org/ 10.1038 /nature 11537

Boussif, O., Lezoualc'h, F., Zanta, M. A., Mergny, M. D., Scherman, D., Demeneix, B., \& Behr, J. P. (1995). A versatile vector for gene and oligonucleotide transfer into cells in culture and in vivo: Polyethylenimine. Proceedings of the National Academy of Sciences of the United States of America, 92, 7297-7301. http://dx.doi.org/10.1073/pnas.92.16.7297

Cong, L., Ran, F. A., Cox, D., Lin, S., Barretto, R., Habib, N., ... Zhang, F. (2013). Multiplex genome engineering using CRISPR/Cas systems. Science, 339, 819-823. http://dx.doi.org/10.1126/science.1231143

Dupuy, A. J., Clark, K., Carlson, C. M., Fritz, S., Davidson, A. E., Markley, K. M., ... Largaespada, D. A. (2002). Mammalian germ-line transgenesis by transposition. Proceedings of the National Academy of Sciences of the United States of America, 99, 4495-4499. http://dx.doi.org/10.1073/pnas.062630599

Fischer, D., Bieber, T., Li, Y., Elsässer, H. P., \& Kissel, T. (1999). A novel non-viral vector for DNA delivery based on low molecular weight, branched polyethylenimine: Effect of molecular weight on transfection efficiency and cytotoxicity. Pharmaceutical Research, 16, 1273-1279. http://dx.doi.org/10.1023/A:101486 1900478

Huh, S. H., Do, H. J., Lim, H. Y., Kim, D. K., Choi, S. J., Song, H., .. Kim, J. H. (2007). Optimization of 25 $\mathrm{kDa}$ linear polyethylenimine for efficient gene delivery. Biologicals, 35, 165-171. http://dx.doi.org/10.1016/ j.biologicals.2006.08.004

Jordan, B. J., Vogel, S., Stark, M. R., \& Beckstead, R. B. (2014). Expression of green fluorescent protein in the chicken using in vivo transfection of the piggyBac transposon. Journal of Biotechnology, 173, 86-89. http://dx.doi.org/10.1016/j.jbiotec.2014.01.016

Kang, K. S., Lee, H. C., Kim, H. J., Lee, H. G., Kim, Y. M., Lee, H. J., ... Han, J. Y. (2015). Spatial and temporal action of chicken primordial germ cells during initial migration. Reproduction, 149, 179-187. http://dx.doi.org/10.1530/REP-14-0433

Kawakami, K., Shima, A., \& Kawakami, N. (2000). Identification of a functional transposase of the Tol2 element, an Ac-like element from the Japanese medaka fish, and its transposition in the zebrafish germ lineage. Proceedings of the National Academy of Sciences of the United States of America, 97, 11403-8. http://dx.doi.org/10.1073/pnas.97.21.11403

Kim, Y. G., Cha, J., \& Chandrasegaran, S. (1996). Hybrid restriction enzymes: Zinc finger fusions to Fok I cleavage domain. Proc Natl Acad Sci, USA, 93, 1156-1160. http://dx.doi.org/10.1073/pnas.93.3.1156

Kong, B.-W., Carlson, D. F., Fahrenkrug, S. C., \& Foster, D. N. (2008). Application of the Sleeping Beauty transposon system to avian cells. Animal Genetics, 39, 180-186. http://dx.doi.org/10.1111/j.1365-2052. 2008.01702.x

Lillico, S. G., McGrew, M. J., Sherman, A., \& Sang, H. M. (2005). Transgenic chickens as bioreactors for protein-based drugs. Drug Discovery Today. http://dx.doi.org/10.1016/S1359-6446(04)03317-3

Lillico, S. G., Sherman, A., McGrew, M. J., Robertson, C. D., Smith, J., Haslam, C., ... Sang, H. M. (2007). Oviduct-specific expression of two therapeutic proteins in transgenic hens. Proceedings of the National Academy of Sciences of the United States of America, 104, 1771-1776. http://dx.doi.org/10.1073/ pnas.0610401104

Liu, X., Li, N., Hu, X., Zhang, R., Li, Q., Cao, D., ... Liu, X. (2012). Efficient production of transgenic chickens based on piggyBac. Transgenic research, 22, 417-423. http://dx.doi.org/10.1007/s11248-012-9642-y

Lu, S., Morris, V. B., \& Labhasetwar, V. (2015). Codelivery of DNA and siRNA via arginine-rich PEI-based polyplexes. Molecular Pharmaceutics, 12, 621-629. http://dx.doi.org/10.1021/mp5006883 
Lungwitz, U., Breunig, M., Blunk, T., \& Göpferich, A. (2005). Polyethylenimine-based non-viral gene delivery systems. European Journal of Pharmaceutics and Biopharmaceutics, 247-266. http://dx.doi.org/10.1016/ j.ejpb.2004.11.011

Macdonald, J., Taylor, L., Sherman, A., Kawakami, K., Takahashi, Y., Sang, H. M., \& McGrew, M. J. (2012). PNAS Plus: Efficient genetic modification and germ-line transmission of primordial germ cells using piggyBac and Tol2 transposons. Proceedings of the National Academy of Sciences, 109, E1466-E1472. http://dx.doi.org/10.1073/pnas.1118715109

Mates, L., Chuah, M. K., Belay, E., Jerchow, B., Manoj, N., Acosta-Sanchez, A., ... Izsvak, Z. (2009). Molecular evolution of a novel hyperactive Sleeping Beauty transposase enables robust stable gene transfer in vertebrates. Nature Genetics, 41, 753-761. http://dx.doi.org/10.1038/ng.343

McGrew, M. J., Sherman, A., Ellard, F. M., Lillico, S. G., Gilhooley, H. J., Kingsman, A. J., ... Sang, H. (2004). Efficient production of germline transgenic chickens using lentiviral vectors. EMBO Reports, 5, 728-733. http://dx.doi.org/10.1038/sj.embor.7400171

Morishita, Y., Imai, T., Yoshizawa, H., Watanabe, M., Ishibashi, K., Muto, S., \& Nagata, D. (2015). Delivery of microRNA-146a with polyethylenimine nanoparticles inhibits renal fibrosis in vivo. International Journal of Nanomedicine, 10, 3475-3488. http://dx.doi.org/10.2147/IJN.S82587

Park, T. S., \& Han, J. Y. (2012). piggyBac transposition into primordial germ cells is an efficient tool for transgenesis in chickens. Proceedings of the National Academy of Sciences, 109, 9337-9341. http://dx.doi.org/10.1073/pnas.1203823109

Rashidi, H., \& Sottile, V. (2009). The chick embryo: Hatching a model for contemporary biomedical research. BioEssays. http://dx.doi.org/10.1002/bies.200800168

Sasakura, Y., Awazu, S., Chiba, S., \& Satoh, N. (2003). Germ-line transgenesis of the Tc1/mariner superfamily transposon Minos in Ciona intestinalis. Proceedings of the National Academy of Sciences of the United States of America, 100, 7726-7730. http://dx.doi.org/10.1073/pnas.1230736100

Sato, Y., Kasai, T., Nakagawa, S., Tanabe, K., Watanabe, T., Kawakami, K., \& Takahashi, Y. (2007). Stable integration and conditional expression of electroporated transgenes in chicken embryos. Developmental Biology, 305, 616-624. http://dx.doi.org/10.1016/j.ydbio.2007.01.043

Scott, B. B., Velho, T. A., Sim, S., \& Lois, C. (2010). Applications of avian transgenesis. ILAR Journal, 51, 353-361.

Sramkova, M., Parente, L., Wigand, T., Aye, M.-P., Shitara, A., \& Weigert, R. (2014). Polyethyleniminemediated expression of transgenes in the acinar cells of rats salivary glands in vivo. Frontiers in Cell and Developmental Biology, 2, 74. http://dx.doi.org/10.3389/fcell.2014.00074

Stern, C. D. (2005). The chick: A great model system becomes even greater. Developmental Cell, 8, 9-17. http://dx.doi.org/10.1016/j.devcel.2004.11.018

Sumiyama, K., Kawakami, K., \& Yagita, K. (2010). A simple and highly efficient transgenesis method in mice with the Tol2 transposon system and cytoplasmic microinjection. Genomics, 95, $306-311$. http://dx.doi.org/10.1016/j.ygeno.2010.02.006

Tang, M. X., \& Szoka, F. C. (1997). The influence of polymer structure on the interactions of cationic polymers with DNA and morphology of the resulting complexes. Gene Therapy, 4, 823-832. http://dx.doi.org/10.1038/sj.gt.3300454

Tyack, S. G., Jenkins, K. A., O’Neil, T. E., Wise, T. G., Morris, K. R., Bruce, M. P., ... Doran, T. J. (2013). A new method for producing transgenic birds via direct in vivo transfection of primordial germ cells. Transgenic Research, 22, 1257-1264. http://dx.doi.org/10.1007/s11248-013-9727-2

Vergara, M. N., \& Canto-Soler, M. V. (2012). Rediscovering the chick embryo as a model to study retinal development. Neural Development, 7, 22-40. http://dx.doi.org/10.1186/1749-8104-7-22

Watanabe, M., Naito, M., Sasaki, E., Sakurai, M., Kuwana, T., \& Oishi, T. (1994). Liposome-mediated DNA transfer into chicken primordial germ cells in vivo. Molecular Reproduction and Development, 38, $268-274$. http://dx.doi.org/10.1002/mrd.1080380307

Wilber, A., Frandsen, J. L., Geurts, J. L., Largaespada, D. A., Hackett, P. B., \& McIvor, R. S. (2006). RNA as a source of transposase for Sleeping Beauty-mediated gene insertion and expression in somatic cells and tissues. Molecular Therapy, 13, 625-630. http://dx.doi.org/10.1016/j.ymthe.2005.10.014 
Yusa, K., Zhou, L., Li, M. A., Bradley, A., \& Craig, N. L. (2011). A hyperactive piggyBac transposase for mammalian applications. Proceedings of the National Academy of Sciences of the United States of America, 108, 1531-1536. http://dx.doi.org/10.1073/pnas.1008322108

\section{Copyrights}

Copyright for this article is retained by the author(s), with first publication rights granted to the journal.

This is an open-access article distributed under the terms and conditions of the Creative Commons Attribution license (http://creativecommons.org/licenses/by/4.0/). 